\title{
A Model-based Music Recommender System using Collaborative Filtering Technique
}

\author{
Adi Riyan Prasetya \\ Faculty of Computer Science \\ Universitas Mercu Buana \\ Jakarta, Indonesia
}

\author{
Desi Ramayanti \\ Faculty of Computer Science \\ Universitas Mercu Buana \\ Jakarta, Indonesia
}

\begin{abstract}
Collaborative filtering (CF) techniques have been proven to be one of the successful approaches to building music recommender systems (MRS). The CF's system work by creates suggestions for users based on their neighbors preferences. On the other hand the online music sites data are highly dynamic. As a result, the listeners face the problem of missing music suggestions. Recommender systems help the users to find their music interests and information they are looking for. In this paper we provide other researchers in that field with new valuable knowledge and insights regarding music recommendations using collaborative filtering technique that has the ability to recommend music to a new user as well as the other existing users. We then present three main categories of collaborative filtering techniques: memorybased, model-based, and the last hybrid method that combining collaborative and content-based.
\end{abstract}

\section{General Terms}

Recommender System, Collaborative Filtering.

\section{Keywords}

Music Personalization, Music Recommendation System.

\section{INTRODUCTION}

Music sites such as Spotify or Apple Music been gaining momentum over the last decade and offer unlimited access to large catalogues of music. These providers store the music in a server that users can connect to via their computers and mobile devices. The increasing competition on the online music sites has forced these businesses to improve their techniques and their online environment to target their customers accurately and to maintain a high level of satisfaction. While the number of music available online is excessively large, online music services now aim at instantly providing their customers with the music content they are seeking, reducing the efforts of their customers as much as possible. Consequently, the importance of music recommender systems can be considered a way of combining these aspects in order to help consumers find the information or something they would be interested in.

Recommender systems have become increasingly relevant with the advent of online music sites, which have made music more accessible. Music recommender is there to help users to filter and discover songs according to their taste, age, region and language[1]. This system collects relevant data about its users and applies mathematical models and diverse techniques to find and propose items[2], which might be of interest for the listener. Initially different recommendation systems constructed with different methods implementing to provide recommendations to users[3]. The benefits that music recommender systems can bring to online music platforms are widely recognized and help users discover new music by providing recommendations, for example, in the section of automatically generated playlists containing music tracks the recommender system thinks the user might like. Recommender systems are now an integral part of e-business sites[4][5]. Some of the music sites that use recommenders as examples, Amazon.com[6], the largest online retailer in the world and Netflix.com[7], the largest online distributor of streaming media, are two well-known and successful websites that established efficient product recommenders following collaborative filtering methods.

\section{RELATED WORK}

In this section, we explain collaborative filtering system, such details as recommender systems, and methods of selecting collaborative filtering systems that are required for the recommendation of music.

\subsection{Recommender System}

Recommender System uses two types of feedback mechanism based on explicit rating and implicit rating[8]. There's two part which recommendation system consists [9]:

\section{- Collaborative filtering}

- Content Based Filtering.

Most of RS system is to generate meaningful recommendations to a collection of users for items or products that might interest them. Their priority is to create and examine user's individual profiles, which contain their preferences, then update the service content to finally increase the user's satisfaction.

\subsection{Collaborative Filtering}

Collaborative filtering is the most common method for recommender and predicts user preferences for items based on the ratings or behavior of others users in the system. This technique assumes a list of $m$ users $U=\{u 1, u 2, \ldots, u m\}$ and a list of $n$ items $I=\{i 1, i 2, \ldots, i n\}$. Each user ui has rated a list of items noted by Iui. The purpose of this technique is to predict the ratings of unrated items by a given user and recommend the Top- $\mathrm{N}$ items[10]. Most recommendation systems applied collaborative filtering methods or contentbased techniques to recommend items of interest to the users[11][12]. Collaborative Filtering can further be divided into three types:

\subsubsection{Memory-based}

Memory-based has proven to be successful in various types of personalized recommender systems[13]. Memory-based refers to any algorithm that relies on a mapping to and from memory. It can be used for recommender algorithms and predicts items based on the complete collection of previous ratings, with algorithms such as the clustering[14], neighborhood based filtering and item-based top-N recommendations.

\subsubsection{Model-based uses data mining}


This method to train and model user's preferences, then makes prediction for test based on the known model. According to Tarang Raval \& Yask patel [15] propose a new hybrid based recommendation method that applying association mining algorithm for rules generation and based on the rules generated will try to generate strong rules and with the help of strong rules best items will be recommended to the user.

\subsubsection{Hybrid method}

The last method that combining collaborative and contentbased recommendation can be more effective and is therefore called hybrid method, which out performs both individual models. The Hybrid methods combine other filtering techniques to achieve better recommendations.

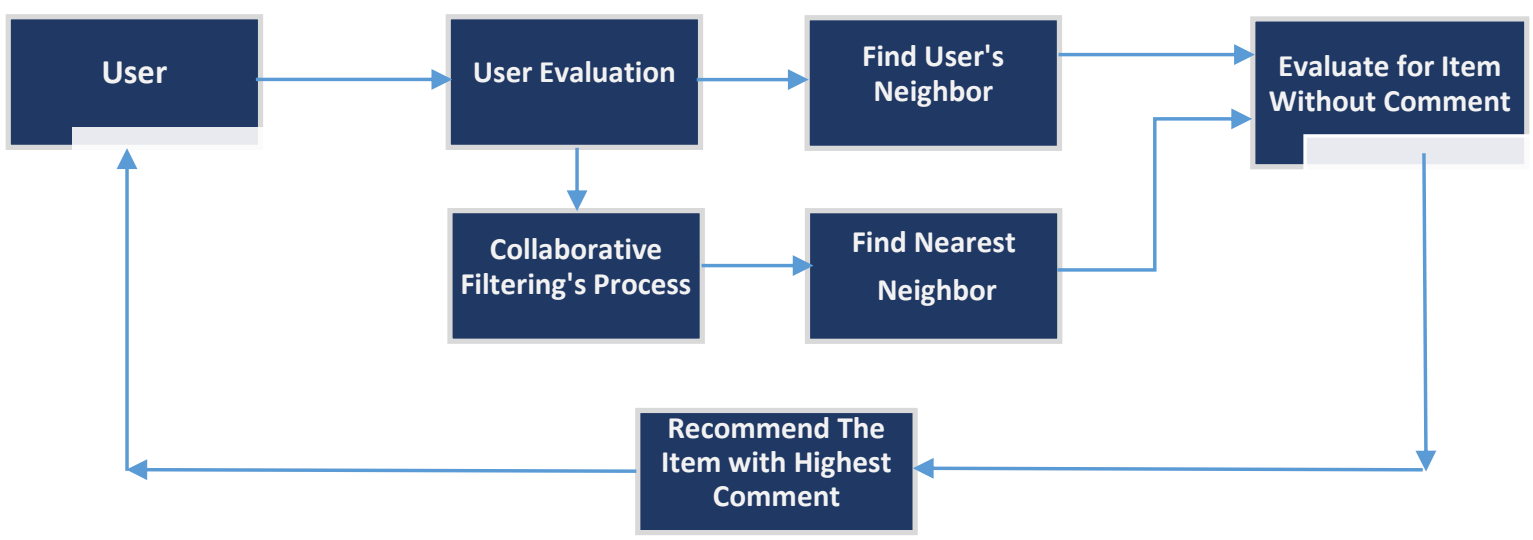

\section{Fig.1 Collaborative Filtering Recommendation}

In Figure 1, the produce of collaborative filtering is illustrated in a flowchart. It can be seen as a loop, where the user gets the recommended item with the highest comment in result of user's evaluation and finding the neighbor. This circulation should run continuously to improve the recommendations, since it takes time. Based on the data and user's interests, neighbor user's are being searched who share the similar interests. The system then recommends the neighbor user's music to the user.

\subsection{Content Based}

In content-Based recommendations depends on users former choices[16]. It recommends or analyzes items based on the items which are highly rated and similar to the users preferences[17]. Content based filtering algorithms are based on the description of an item refer to content whose attributes are used in the recommender models. Thus, content based filtering algorithm suggest items that are similar to the items which are liked by the user in the past.

\section{PROPOSED WORK}

In other words, the recommendation mechanisms predicts the relevance between the users and items and thereby generates a ranking list of items[18]. For example a taste profile will be developed for each user with the individual taste in music. It's created based on the frequency of playing to a song, whether the user skipped tracks or not which works as thumbs up and down and with explorations. In this selection, we give a brief the technical approach of collaborative filtering with Self Information equations below (1).

$\frac{\operatorname{Min}}{\mathcal{H} * \gamma *} \sum_{u, i} C_{u}\left(P u i-x_{u}^{T} y_{i}\right)^{2}+\left(\sum_{u}\left\|x_{u}\right\|^{2}+\sum_{i}\left\|\gamma_{i}\right\|^{2}\right)$

The variable "Pui" probability of exposure to an item $i$ for an user $\mathrm{u}$, where the user likes the item by consuming the item once, when "Pui" equals one. Otherwise the user never consumed the item before. There are different levels of confidence within items, but for easier illustration we use the binary variable. The next variable "Cui" measures the confidence depending on "pui". The aim however is to find a vector " $X u$ " for user " $u$ " and "Y $i$ " for item " $i$ " that will factor user preferences. The calculation are similar with matrix factorization technique. In the last term is for regularizing the model to avoid over fitting of training data.

Table 1. Music data set

\begin{tabular}{|c|c|c|c|}
\hline artist_name & song_id & song_title & total_play \\
\hline Artist A & 001 & A & 5 \\
\hline Artist B & 002 & B & 3 \\
\hline Artist C & 003 & C & 3 \\
\hline Artist D & 004 & D & 1 \\
\hline Artist X & $00 X$ & X & 7 \\
\hline
\end{tabular}

$$
\begin{aligned}
& x_{u}=\left(\mathcal{Y}^{t} C^{u} Y+\lambda I\right)^{-i} Y^{t} C^{u} p(u) \\
& y_{i}=\left(X^{t} T^{i} X+\lambda I\right)^{-i} X^{t} C^{i} p(i)
\end{aligned}
$$

This model leads to an alternating least squares optimization process, where the item and user vectors are initialized at the beginning. Then the item vectors are fixed and solved for optimal user vectors, by taking the derivate of the min function, setting equal zero and solving (2). The user vectors are fixed and solved for optimal item vectors (3). This procedure will be repeated until it converges.

Table 2. Training data song from user

\begin{tabular}{|c|c|c|c|}
\hline \multirow{7}{*}{ user_id } & artist_name & song_title & total_play \\
\cline { 2 - 4 } & Artist A & A & 75 \\
\cline { 2 - 4 } & Artist X & X & 63 \\
\cline { 2 - 4 } & Artist B & B & 30 \\
\cline { 2 - 4 } & Artist D & D & 17 \\
\cline { 2 - 4 } & Artist C & C & 15 \\
\hline
\end{tabular}




\section{EXPERIMENTS RESULT}

Following (figure 2) the feedback is loop, where the collected output information is sent back to the input and adjusts by the behaviors.

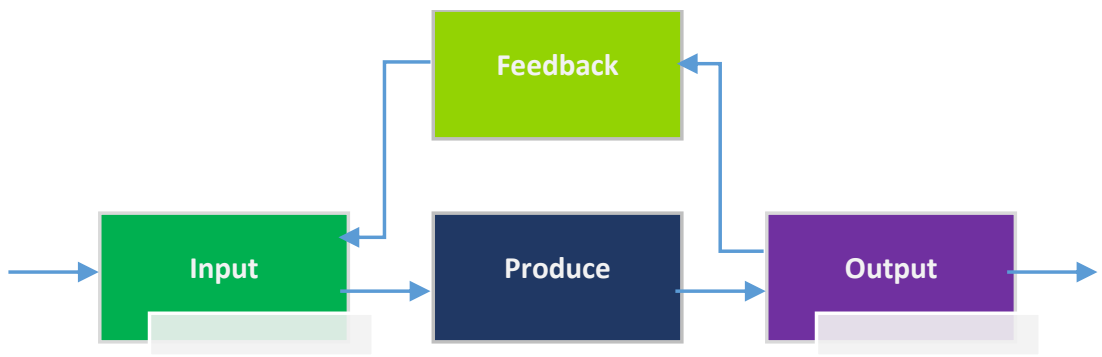

Fig.2 General system theory with feedback.

These process is called feedback control by error, because the system controls the behavior and eliminates errors. As the name can tell, the system detects and analyses information after a learning research and reflects the given information. The characteristics features of feedback shall contain to process information timely and accurately, be helpful for predicting and to control plans and management. So it refers to the output of the system but also returns to the input to change information to influence operations. Other than that the feedback system needs features such as targeted and continuity, requirements e.g. true and valid information and minimize time and last but not least development, where the system has to be sensitive, correct and strong.

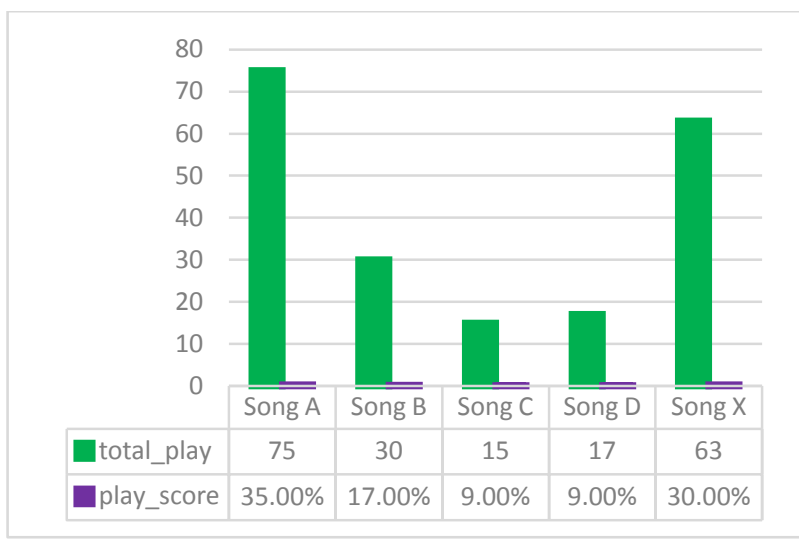

Fig.3 Frequency of play when songs matching user tastes.

Figure 3. The following chart, user are play or add the music into their playlist is widely accepted. The RS's service is based on the history of plays record. As shown in the figure above, 9 percent barely click "play". However less than a third have the habit to play or add music on their playlist every time, and another 9 percent want to adopt this habit.

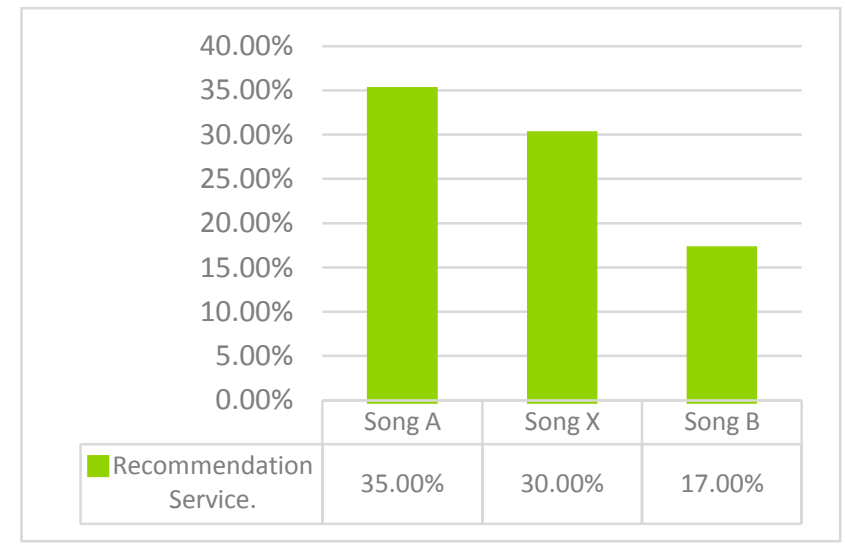

Fig.4 System recommended music to the user.

Figure 4 . The songs with highest rate will be recommended to the other users in the future. The question was whether the recommended music would match the taste of the users or not. And the majority of people did not get a customized recommendation while listening to music. Only less than 40 per cent are fully satisfied with the recommendation service. So there is still progress to make for the feedback system.

\section{CONCLUSION}

This chapter has introduced the main collaborative filtering techniques that can be applied in the design of a music recommender system. We have also surveyed their use in the literature and provided some rough guidelines on how and where they can be applied. Beginning with the disadvantages of Collaborative Filtering, collectively are referred to as the Cold-start problem. Most users do not rate most items and hence the user ratings matrix is typically very sparse, since it decreases the probability of finding a set of users with similar ratings where an item cannot be recommended unless some user has rated it before. As advantages, Collaborative Filtering its predictions and recommendations on the ratings of other users in the system and evaluates information that is difficult to be analyzed. It also avoids low accuracy by matching items with neighbor-hood users and provides the users with not similar recommendations but based on the user's taste. To summarize the feedback system, the main disadvantage is the problem of time delay and transient behavior of the process can be easily manipulated, and there still need to be the right features and development for every feedback system. 


\section{REFERENCES}

[1] B. Dey, "Music-Map: A Music Recommendation System Based On Data Mining Technique," Ver. XI.

[2] P. No, F. Ahmad, and A. K. M, "Available Online at www.journalijcar.org Review Article REVIEW BASED RECOMMENDER SYSTEM FOR BUSINESS ADVANTAGE USING NATURAL LANGUAGE PROCESSING," vol. 7, no. 5, 2018.

[3] M. Nilashi, K. Bagherifard, O. Ibrahim, and H. Alizadeh, "Collaborative Filtering Recommender Systems Collaborative Filtering Recommender Systems," no. September 2016, 2013.

[4] V. Manvitha and M. S. Reddy, "Music Recommendation System Using Association Rule Mining and Clustering Technique To Address Coldstart Problem," vol. 3, no. 7, pp. 6855-6858, 2014.

[5] M. B. Dias, D. Locher, B. Dias, D. Locher, and W. Elderedy, "The Value of Personalised Recommender Systems to e-Business: A Case Study Categories and Subject Descriptors," no. January, 2008.

[6] N. Abdullah, Y. Xu, and S. Geva, "Integrating collaborative filtering and matching-based search for product recommendations," J. Theor. Appl. Electron. Commer. Res., 2013.

[7] C. A. Gomez-uribe and N. Hunt, "The Netflix Recommender System: Algorithms , Business Value ," vol. 6, no. 4, 2015.

[8] M. Misal and U. Nagaraj, "Recommender System Methods and Feedback Mechanisms: A Survey," pp. 11710-11716, 2015

[9] T. Sultana, N. Sharma, S. Aher, N. Pate, and M. Kinikar, "Recommendation System for Music File."
[10] G. Badaro, H. Hajj, W. El-hajj, and L. Nachman, "A Hybrid Approach with Collaborative Filtering for Recommender Systems A Hybrid Approach with Collaborative Filtering for Recommender Systems," no. July, 2013.

[11] C. Castelino, R. S. Muthalaly, A. Lopes, and S. Lopes, "Recommender Systems: Increasing Profits and Efficiency in Business," Int. J. Eng. Trends Technol., vol. 16,2014

[12] K. Choi, Y. Suh, and D. Yoo, "Extended Collaborative Filtering Technique for Mitigating the Sparsity Problem Previous works," vol. 11, no. October, pp. 631-644, 2016.

[13] K. Yu, A. Schwaighofer, V. Tresp, X. Xu, and H. P. Kriegel, "Probabilistic Memory-Based Collaborative Filtering,” IEEE Trans. Knowl. Data Eng., 2004.

[14] A. F. Huda, I. Wasito, T. Basaruddin, and S. Mujiono, "Spatial Clustering Algorithm Based on Neighboring Structure Approach," vol. 8, no. November, pp. 25-38, 2013.

[15] M. S. Reddy, "User Based Collaborative Filtering for Music Recommendation System Abstract:," vol. 2, no. 12, pp. 185-190, 2013.

[16] P. B. Thorat, "Survey on Collaborative Filtering , Content-based Filtering and Hybrid Recommendation System," vol. 110, no. 4, pp. 31-36, 2015.

[17] N. Pereira and S. Varma, "Survey on Content Based Recommendation System.

[18] G. Advani, "A Novel Method for Music Recommendation using Social Media Tags," vol. 122, no. 2 , pp. 37-43, 2015 . 